Received: 11 November 2018

Accepted: 16 April 2019

Published online: 24 April 2019

ENTIFIC REPRTS

\title{
Accurate early prediction of tumour response to PDT using optical coherence angiography
}

M. A. Sirotkina ${ }^{1}$, A. A. Moiseev ${ }^{2}$, L. A. Matveev ${ }^{2}$, V.Y. Zaitsev², V. V. Elagin $\mathbb{1}^{1}$, S. S. Kuznetsov' ${ }^{1}$, G. V. Gelikonov' ${ }^{2}$, S. Y. Ksenofontov ${ }^{2}$, E. V. Zagaynova ${ }^{1}$, F. I. Feldchtein ${ }^{1}$, N. D. Gladkova ${ }^{1} \&$ A. Vitkin ${ }^{3}$

Prediction of tumour treatment response may play a crucial role in therapy selection and optimization of its delivery parameters. Here we use optical coherence angiography (OCA) as a minimally-invasive, label-free, real-time bioimaging method to visualize normal and pathological perfused vessels and monitor treatment response following vascular-targeted photodynamic therapy (PDT). Preclinical results are reported in a convenient experimental model (CT-26 colon tumour inoculated in murine ear), enabling controlled PDT and post-treatment OCA monitoring. To accurately predict long-term treatment outcome, a robust and simple microvascular metric is proposed. It is based on perfused vessels density (PVD) at $\mathrm{t}=\mathbf{2 4}$ hours post $\mathrm{PDT}$, calculated for both tumour and peri-tumour regions. Histological validation in the examined experimental cohort $(n=31$ animals) enabled further insight into the excellent predictive power of the derived early-response OCA microvascular metric. The results underscore the key role of peri-tumour microvasculature in determining the long-term PDT response.

Local treatment, good cosmetic outcome, low normal tissue toxicity and ability to retreat if necessary make photodynamic therapy (PDT) very attractive for treatment of small solid tumours ${ }^{1-4}$. PDT's mechanism of action is based on light activation of photosensitizer molecules and formation of singlet oxygen in conditions of good tissue blood supply and oxygenation ${ }^{5}$. Various photosensitizer formulations have been proposed to enhance their preferential accumulation in the tumour tissues, enhancing PDT's efficacy, minimal side effects and good cosmetic outcomes ${ }^{5}$.

Uptake of the photosensitizer into cancer regions, spatial 3D distribution of the activating light, and oxygen tension in the irradiated tissue volumes all contribute to treatment efficacy ${ }^{6,7}$. Appropriate control of these three factors is necessary to ensure successful PDT outcomes. However, measurement of all these parameters is challenging in clinical conditions, and their significant spatio-temporal variations make prediction of PDT efficacy in individual patients very difficult ${ }^{8}$.

Various treatment planning, dosimetry, monitoring and feedback strategies have been proposed to optimize and improve PTD efficacy. Broadly, the 'explicit' (e.g., singlet oxygen luminescence monitoring) 9,10 $^{10}$ and 'implicit' (e.g., photosensitizer photobleaching measurements) ${ }^{11,12}$ approaches to PDT dosimetry have been investigated. Despite the high sensitivity and direct detection of the resultant PDT "active agent", the technical complexity and limitations of explicit methods have resulted in their limited clinical use ${ }^{9,10,13}$. Analogously, the implicit PDT dosimetry, specifically based on photosensitizer photobleaching, seems to be only loosely related to long-term clinical outcome $\mathrm{e}^{14,15}$.

Given the importance of microvasculature as the means of tissue oxygen supply and as a potential target of PDT, angiographic imaging may offer a promising avenue for monitoring PDT response. With its excellent 3D spatial resolution, fast imaging speed, noninvasive nature and various contrast-free means of detecting blood microcirculation, optical coherence tomography (OCT) appears well suited to this task ${ }^{14}$. The various OCT vessels-contrasting methods that have been developed include Doppler OCT ${ }^{16}$, speckle variance OCT ${ }^{17}$ and phase variance $\mathrm{OCT}^{18}$, with corresponding pros/cons with respect to microvascular detectivity, robustness to bulk tissue

${ }^{1}$ Privolzhsky Research Medical University, Minin Square 10/1, 603950, Nizhny Novgorod, Russia. ${ }^{2}$ Institute of Applied Physics of the Russian Academy of Sciences, Ulyanov Street 46, 603950, Nizhny Novgorod, Russia. ${ }^{3}$ Departments of Medical Biophysics and Radiation Oncology, University of Toronto and University Health Network, 610 University Ave., Toronto, Ontario, M5G 2M9, Canada. Correspondence and requests for materials should be addressed to M.A.S. (email: sirotkina_m@mail.ru) 
motion, vessel shadowing artefacts, flow detection versus microvessel network visualization, and so forth ${ }^{19,20}$. The optical coherence angiography (OCA) variant we employ here ${ }^{21}$ enables sensitive 3D detection of microvessels that are actively perfused with flowing blood, thus furnishing microstructural and functional volumetric microcirculation images, as is important in monitoring PDT processes where perfusion plays the major role. Briefly, our OCA technique is based on high-frequency filtration of partially overlapped OCT data with finite impulse response filter in signal space, allowing real-time visualization of angiography cross-sections to provide useful feedback to the OCT system operator ${ }^{21}$. Our recent research in both preclinical and clinical settings has demonstrated the potential utility of OCA-derived blood perfusion reaction for antitumour therapy monitoring and outcome assessment ${ }^{14,22}$.

As known from literature, tumour vascular damage monitoring during and/or shortly post treatment ( hours) may be useful for prognosis of ultimate tumour reaction to PDT ${ }^{14,23,24}$. For example, our previous study ${ }^{14}$ yielded a simple and convenient criterion of PDT success for the ear tumour model: no perfused vessels on the OCA images at $\mathrm{t}=24$ hours post PDT ${ }^{14}$. However, this was based on longitudinal imaging in a small number of animals $(\mathrm{n}=11)$, and the simple criterion yielded a $20 \%$ false-positive rate; further, PDT damage to the surrounding peri-tumourous microvasculature was not investigated. Indeed, it is well known that tumours were not cured and/or may relapse when the tumour-supplying vasculature in the peri-tumourous tissue was not suitably disabled by $\mathrm{PDT}^{25}$. Considering the potentially important role of tumour-supplying vasculature in treatment outcome, a more comprehensive investigation of PDT effects in both tumour and peri-tumourous blood perfusion in a larger number of animals is thus warranted.

In this study, we report on OCA monitoring of PDT treatments in a preclinical mouse ear model inoculated with CT-26 murine colon tumour cell line ( $\mathrm{n}=31$ animals), a convenient in vivo platform for reproducible tumour growth, controlled PDT delivery, independent tumour volume measurements, and robust OCA monitoring of the relevant tissue volumes. "Mild" PDT irradiation regime was intentionally selected to obtain a spectrum of outcomes that included both responders and non-responders. Outcome predictions based on OCA monitoring of both tumour and near-tumour (peri-tumourous) tissues at several time points following PDT (0-24hrs) were correlated with treatment outcome as revealed by histology at $\mathrm{t}=7$ days post treatment. Microvascular metrics derived from OCA images at $\mathrm{t}=24 \mathrm{hrs}$ were able to accurately distinguish between long-term treatment responders and non-responders, as detailed below.

\section{Results}

Controllable tumour model for OCA-based PDT outcome assessment. Tumour-bearing murine ear model is a convenient controllable in-vivo platform to deliver PDT and quantitatively study resultant microvascular responses. Visual comparison of OCA images of normal and tumour tissues reveals pronounced differences in vascular architecture (Fig. 1a,b) Because of active neovascularization and angiogenesis ${ }^{26}$, the tumour vasculature is denser and appears more tortuous ${ }^{27}$ than in surrounding peri-tumourous tissues (Fig. 1b). The 1-2 mm thickness of the tumour-bearing murine ear preparation permits OCA imaging over the entire tissue depth in peri-tumourous regions, and throughout the majority of the tumour volume, thus enabling detailed 3D microvascular imaging of the relevant tissue responses. The ear model also facilitates independent and accurate tumour volume measurement via calipers, for potential correlation with functional metrics, photobleaching results, and treatment outcome ${ }^{14}$.

OCA visualization of tumour and peri-tumourous PDT effects. Figure 2a demonstrates OCA imaging of tumour and peri-tumourous perfused microvasculature before PDT. For subsequent image quantification, we employ the perfused vessel density (PVD) metric (see subsection Optical coherence angiography in Materials and Methods).

Of the various examined time points post PDT - immediately post, $\mathrm{t}=5 \mathrm{hrs}$ and $\mathrm{t}=24 \mathrm{hrs}$ - the most pronounced vascular reaction of tumour and peri-tumourous tissue vessels was detected by OCA at $\mathrm{t}=24 \mathrm{hrs}$. Figure $2 \mathrm{~b}$ suggests that simultaneous absence of perfused vessels in the tumour site (visually zero PVD) and very low PVD in the peri-tumourous tissue may indicate a successful PDT treatment (responder). Importantly, we further note that animals demonstrating absence of perfused vessels in the tumour itself, but some viable perfused vessels in the peri-tumourous tissue as seen in Fig. 2c, subsequently proved to be non-responders.

Quantification of OCA images for PDT outcome prediction. Quantification and analysis of the obtained 3D microvascular perfusion maps was performed in order to derive OCA biomarkers predictive of PDT outcome. Ideally, use should be made of the earliest possible OCA image sets, when treatment planning/ personalization/correction is most convenient to implement and potentially most impactful. Examining first the pre-PDT data, the perfused vessel densities of both tumour and peri-tumourous tissue did not exhibit a clear correlation with PDT outcome and thus cannot serve as a reliable predictor of the treatment success. Specifically, the tumour PVD before PDT can yield 20 correctly predicted outcomes (CPO) out of 31 cases (70\% accuracy, Wilson score interval $=48-75 \%$ - see Statistical Analysis subsection in Materials and Methods for the threshold PVD selection and Wilson score explanation). Using the peri-tumourous tissue PVD can yield 18 of 31 CPO (58\%, interval $=42-74 \%$ ) (Fig. 3c). It thus appears that pre-treatment microvascular perfusion state cannot be used as a reliable predictor of PDT response.

Not surprisingly, the OCA prediction accuracy improves with the use of post-PDT data, such as immediately following PDT and later (see Fig. 3c). We thus quantified the CPO using the three temporal data sets - immediately post, $\mathrm{t}=5 \mathrm{hrs}$ and $\mathrm{t}=24 \mathrm{hrs}$. The results are summarized in Table 1 , where the pre-treatment numbers discussed above have also been included for completeness.

Several comments can be made about the data trends. First, the present study confirms our previous observation $^{14}$ : only non-responders have perfused vessels on OCA images in the tumour (at $t=24$ hrs post PDT). 


\section{Normal mouse ear}
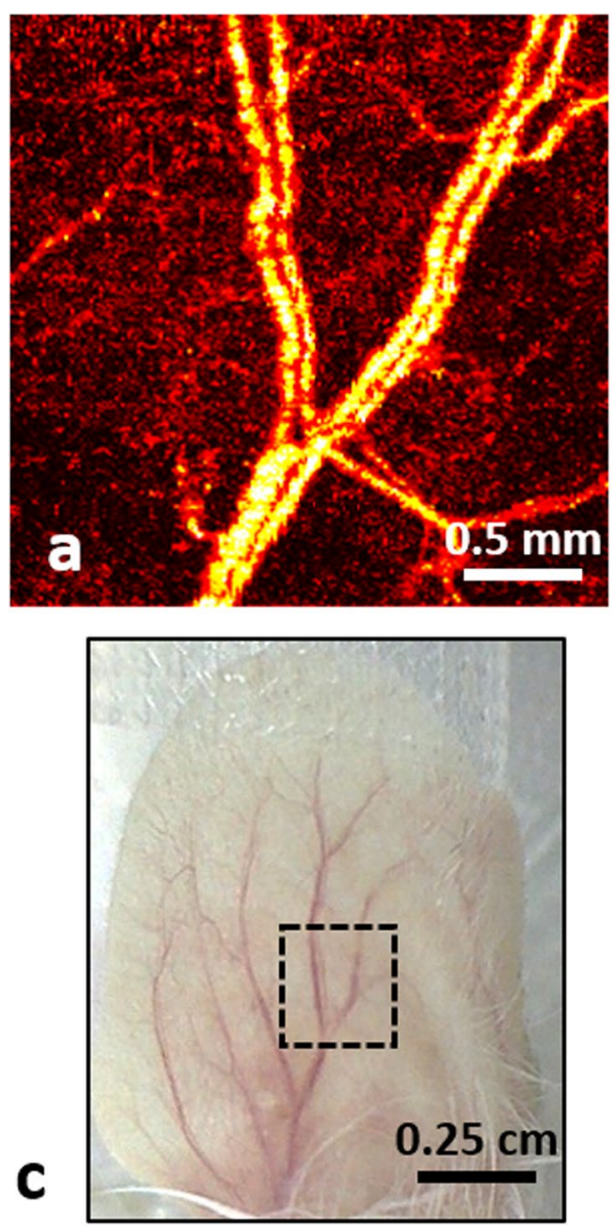

CT-26
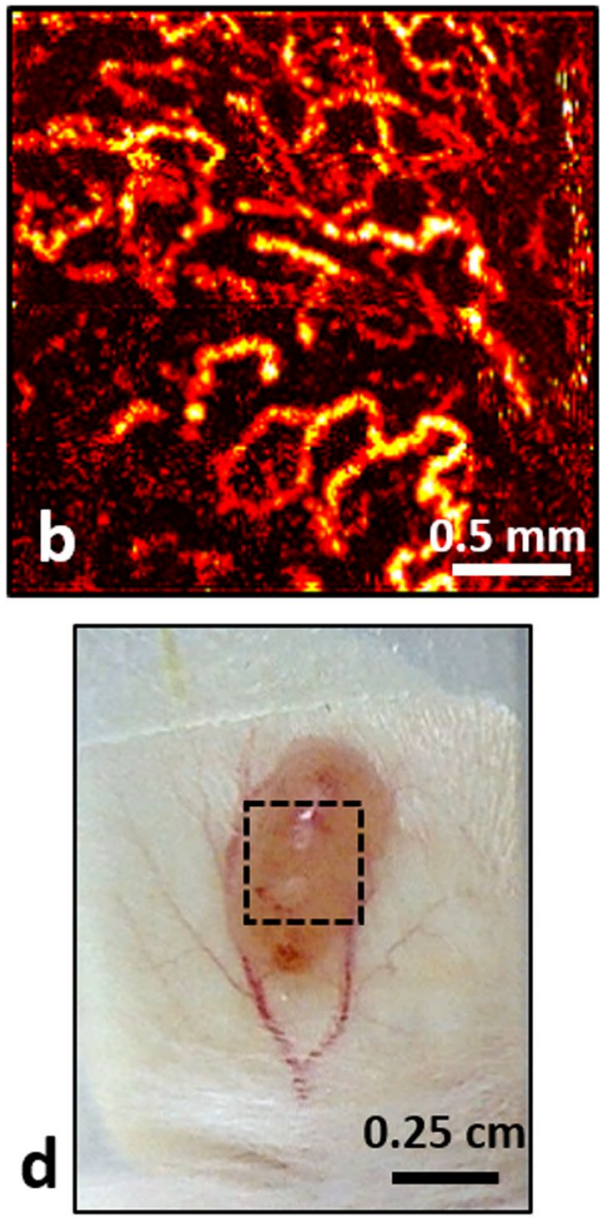

Figure 1. Perfused microvascular network architecture in a mouse ear visualized by optical coherence angiography (OCA). (a) 2D projection of 3D OCA data showing typical normal ear microcirculation; (b) OCA of CT-26 tumour growing in a mouse ear (two weeks after tumour cells inoculation); (c,d) are the corresponding white-light photographs of the mouse ear. Black dotted lines indicate the $2.5 \times 2.5 \mathrm{~mm}^{2}$ regions of OCA imaging.
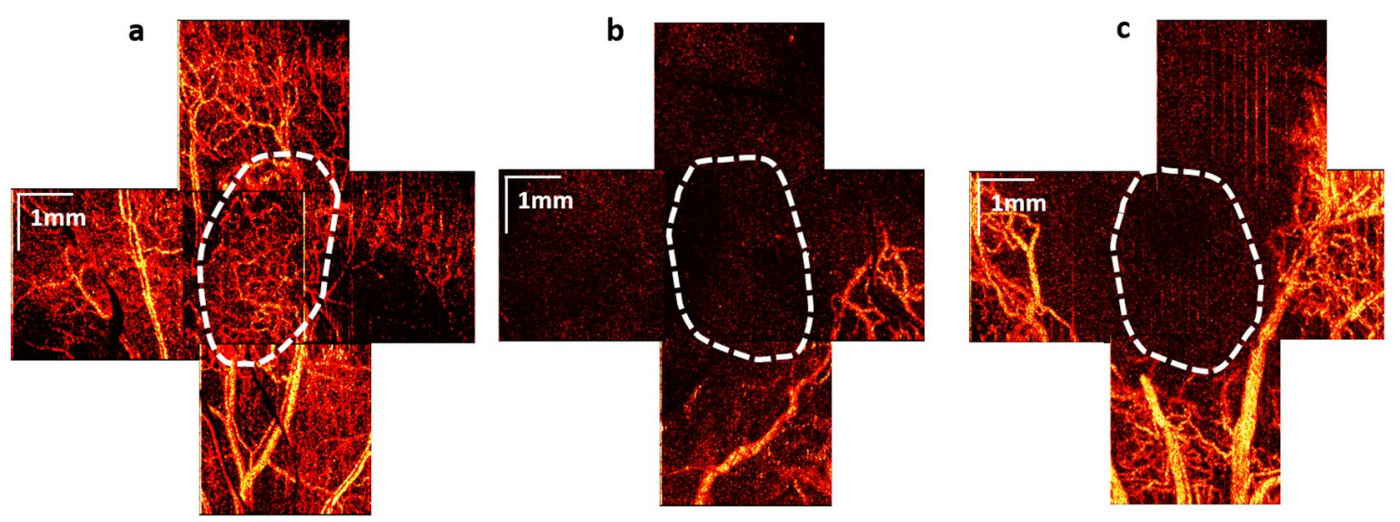

Figure 2. OCA images of tumour and peri-tumourous perfused microvascular reaction at $\mathrm{t}=24 \mathrm{hrs}$ post PDT. (a) before PDT; (b) OCA example of a responder (no visible perfused vessels in the tumour, and extremely low PVD in peri-tumourous tissue); (c) OCA example of a non-responder (no perfused vessels inside the tumour, but many perfused vessels in peri-tumourous tissue). The tumour borders (indicated by dashed contours) were automatically segmented using machine learning pixel classification technique ${ }^{29}$. 

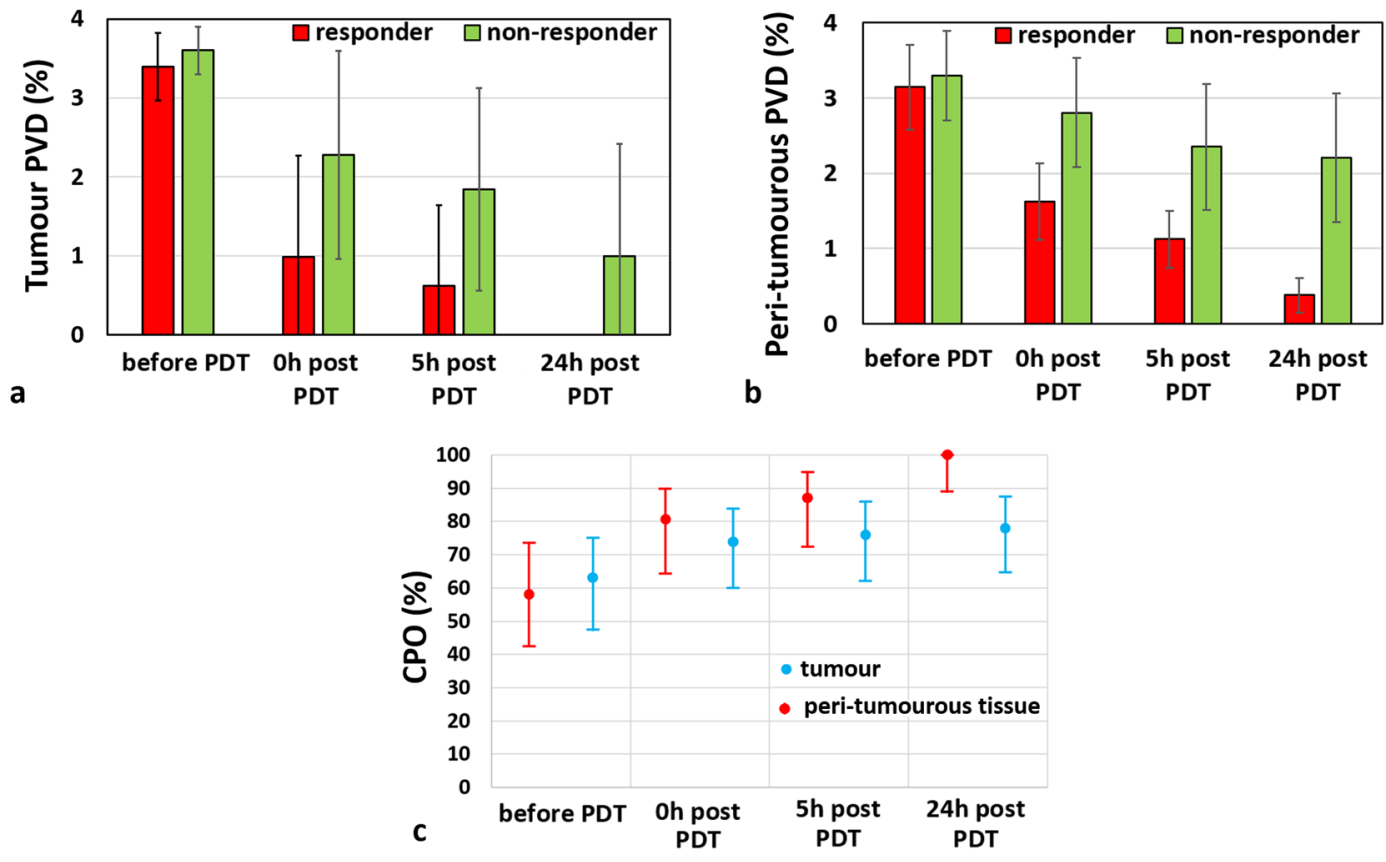

Figure 3. OCA-derived PVD changes after treatment for predicting PDT success. (a) PVD temporal evolution in the tumour, and (b) in the peri-tumourous tissue; error bars signify standard deviation. (c) percentage of correctly predicted outcomes (CPO) based on PVD values at the three post-treatment time points. Dots indicate values calculated from the experimental data, bars indicate the $95 \%$ Wilson score confidence intervals (based on the sample size $(\mathrm{n}=31)$ and the number of correctly predicted outcomes).

\begin{tabular}{|l|l|l|l|l|l|l|l|l|}
\hline Time point & \multicolumn{2}{|l|}{ Before PDT } & \multicolumn{2}{l|}{ 0hrs post PDT } & \multicolumn{2}{l|}{ 5hrs post PDT } & \multicolumn{2}{l|}{ 24hrs post PDT } \\
\hline OCA region & $\begin{array}{l}\text { PVD } \\
\text { tumour }\end{array}$ & $\begin{array}{l}\text { PVD peri- } \\
\text { tumour }\end{array}$ & $\begin{array}{l}\text { PVD } \\
\text { tumour }\end{array}$ & $\begin{array}{l}\text { PVD peri- } \\
\text { tumour }\end{array}$ & $\begin{array}{l}\text { PVD } \\
\text { tumour }\end{array}$ & $\begin{array}{l}\text { PVD peri- } \\
\text { tumour }\end{array}$ & $\begin{array}{l}\text { PVD } \\
\text { tumour }\end{array}$ & $\begin{array}{l}\text { PVD peri- } \\
\text { tumour }\end{array}$ \\
\hline CPO & $21 / 31$ & $19 / 31$ & $23 / 31$ & $25 / 31$ & $24 / 31$ & $27 / 31$ & $23 / 31$ & $31 / 31$ \\
\hline CI $(\%)$ & $48-75$ & $42-74$ & $60-84$ & $64-90$ & $62-87$ & $71-95$ & $64-88$ & $89-100$ \\
\hline Threshold PVD (\%) & 4.0 & 2.7 & 1.45 & 1.85 & 1.12 & 1.83 & 0.22 & 1.0 \\
\hline
\end{tabular}

Table 1. Summary of the obtained numbers of correctly predicted PDT outcomes (CPO row), the corresponding confidence intervals (CI row), and the PVD value thresholds used for separating responders from nonresponders (threshold PVD row) for tumour and peri-tumour PVD at different time points. Threshold PVD value evaluation is described in the Statistical Analysis subsection of the Materials and Methods section.

Thus, if any perfused vessels are visualized within the tumour region, the PDT should be considered unsuccessful (see Fig. 3a). However, the converse is not true - post-treatment absence of perfused vessels inside the tumour is insufficient and itself cannot predict PDT outcome! Indeed, 8 out of 13 non-responders in the present study had no perfused vessels inside the tumour at $\mathrm{t}=24 \mathrm{hrs}$ post PDT. Second, the prediction accuracy improves the longer one waits to collect OCA data post-treatment. This is not surprising, and is seen in the increasing CPO numbers in Table 1 as one moves from pre-treatment to immediately post-treatment data, and further to $t=5 \mathrm{hrs}$ and $\mathrm{t}=24$ hrs data sets.

Examining the $\mathrm{t}=24 \mathrm{hrs}$ data closer, one can note that PVD in the peri-tumourous tissue of responders drops an order of magnitude compared to before PDT ( $0.38 \pm 0.22$ versus $3.14 \pm 0.57 \%)$. In contrast, PVD exhibits only a modest decrease in non-responders $(2.21 \pm 0.86 \%$ versus $3.29 \pm 0.59 \%)$ (see Fig. $3 b)$. These trends suggest the following formulation of the optimal treatment outcome prediction criteria: threshold could be set as an average between the highest PVD value in responders and the lowest PVD value in non-responders groups, yielding a PDV threshold value $=1 \%$ on OCA images. With this thresholding, measurements of peri-tumourous PVD at $\mathrm{t}=24 \mathrm{hrs}$ correctly predict outcome for all 31 examined cases (CPO accuracy $=100 \%$, Wilson score interval $89-100 \%$ ). Of course, this works provided that post-treatment tumour perfusion is minimal (as verified by the $\mathrm{PVD}_{\text {tumour }}$ value being $\sim 0 \%$ ).

To summarize, a simple-to-implement prediction protocol based on $\mathrm{t}=24 \mathrm{hrs}$ post PDT OCA visualization can be formulated. If any perfused vessels are inside the tumour, PDT should be considered unsuccessful. If no perfused vessels are found in OCA images inside tumour borders (so that PDT is potentially successful), then the surrounding tissues in the $\sim 2 \mathrm{~mm}$ proximity should be additionally evaluated. If overall density of perfused vessels in this region does not exceed $1 \%$ on OCA images, PDT can be considered successful. Deviation from this criterion indicate non-responders. These considerations are graphically summarized in the flow chart of Fig. 4. 


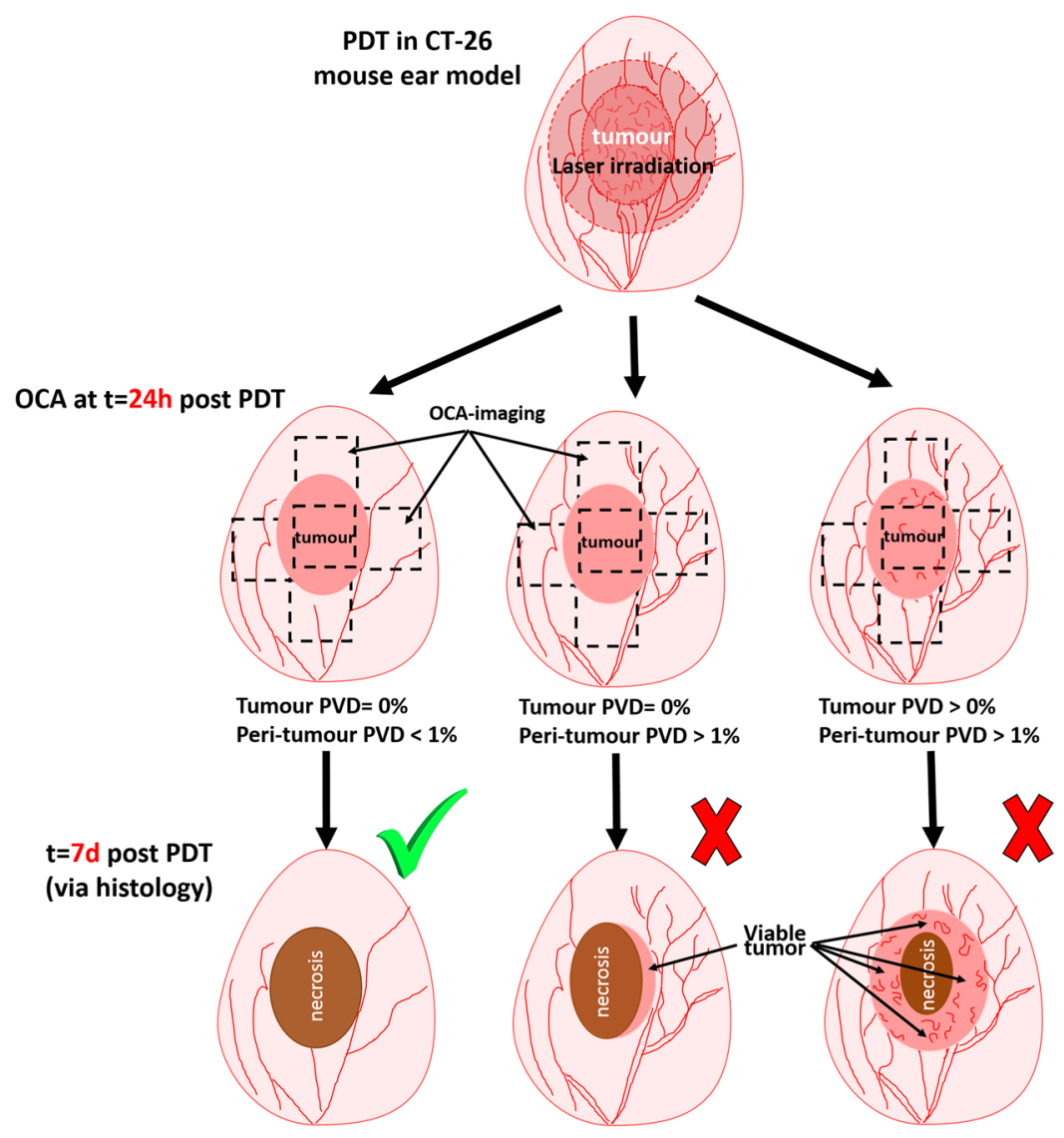

Figure 4. Graphical summary of the OCA-based PDT success assessment scheme, based on tumour and peritumourous microvascular reaction at $\mathrm{t}=24 \mathrm{hrs}$ after treatment.

All OCA-examined animals were subjected to histological assessment at $t=7$ days post PDT, which was used for final treatment outcome determination. As shown in our previous study ${ }^{14}$, we classified responders as having $>95 \%$ total tumour necrosis on histology (Fig. 5a), and non-responders with $<95 \%$ tumour necrosis (Fig. 5d). For PDT responders, peri-tumourous regions including the more distant 'normal' ear tissue (up to $\sim 2 \mathrm{~mm}$ away from the tumour edge) exhibit ischemia, and necrosis is clearly seen on histology (Fig. 5b,c). In cases of non-responders, no damage of peri-tumourous tissue both at the tumour border and $\sim 2 \mathrm{~mm}$ away was histologically visible at 7 days post PDT (Fig. 5e,f). This likely stems from preserved blood perfusion, as seen on OCA images of surviving dense microvascular networks in peri-tumourous tissue at $\mathrm{t}=24 \mathrm{hrs}$ illustrated in Fig. $2 \mathrm{c}$ above. Thus, perfusion preservation (both in the tumour volume or in the pre-tumour regions) can cause treatment failure and/or relapse. Histopathologically-visible necrosis of irradiated tumourous tissue, likely in part due to thrombosis of blood vessels, was observed for both responders and non-responders, suggesting that local tumour response alone is not sufficiently predictive of treatment outcome. This indicates the crucial role of peri-tumourous microvasculature; indeed, peri-tumourous tissues up to $\sim 2 \mathrm{~mm}$ from the pathology edge demonstrated absence of viable perfusion on OCA $(t=24 \mathrm{hrs})$ and yielded histologic necrosis $(\mathrm{t}=7$ days $)$ in PDT success cases.

The control experiments with no-treatment and light-only were performed previously and have been described in detail ${ }^{14}$. Both cohorts demonstrated well-developed tumour blood vessels network on OCA images throughout all observation time period, exhibiting $80-100 \%$ viable tumour cells at the end of the study ${ }^{14}$.

\section{Discussion}

In this study, an accurate early ( $\mathrm{t}=24 \mathrm{hrs}$ post treatment) microvascular perfusion criterion of PDT outcome has been proposed. In addition to local tumour response, the key role of peri-tumourous tissue microvessels in determining treatment success has been experimentally demonstrated. PDT is seen to be successful with the obliteration of tumour perfusion as seen on OCA at $\mathrm{t}=24 \mathrm{hrs}$ post-treatment, and analogous obliteration of peri-tumourous perfusion to a distance of $\sim 2 \mathrm{~mm}$ from the tumour edge. Numerous recent studies, for example by Kutsuyoshi et al. ${ }^{28}$, have analogously demonstrated that blocking both blood circulation routes (tumour and peri-tumourous vessels) has great potential as a clinical strategy to prevent cancer recurrence.

It is known that there are three main mechanisms of PDT action: the vascular, cellular and immune response $\mathrm{e}^{5,6}$. In the present study, PDT was mostly vasculature targeted, but the sensitizer also partially penetrated through the vessels' walls into surrounding tissue, so that cellular and immune responses were involved as well. However, only the vasculature response can be robustly and non-invasively evaluated in real time using the developed OCA. In 
RESPONDER

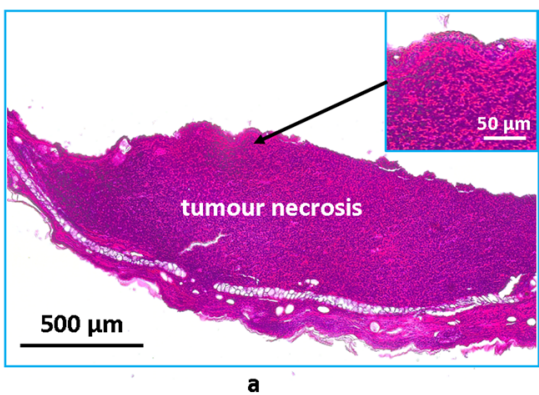

a

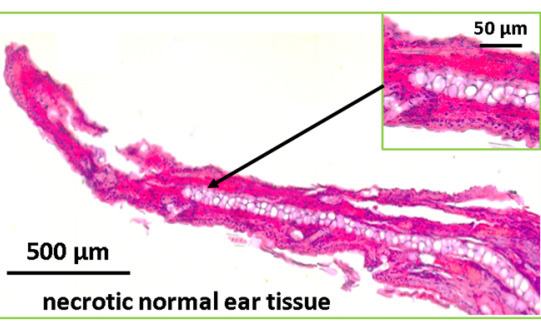

c

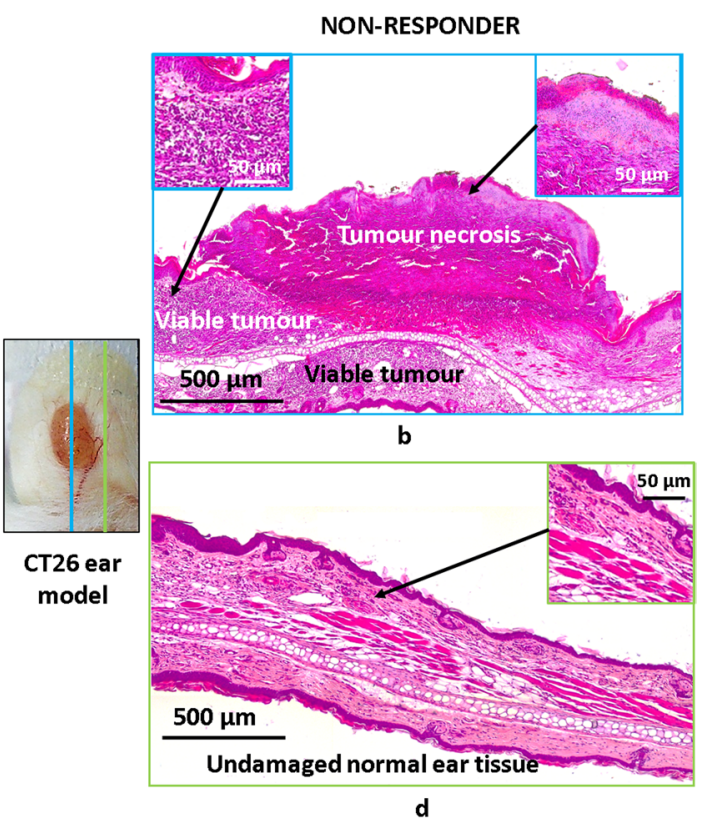

Figure 5. Histological assessment of treatment outcomes at $t=7$ days post PDT. Left column's two panels exhibit an illustrative responder. (a) Total tumour necrosis (>95\%) in the central part of tumour (blue arrows). (b) Total necrosis of 'normal' ear tissue $\sim 2 \mathrm{~mm}$ distant from the tumour edge (but still within the PDT treatment field). Right column's two panels exhibit an illustrative non-responder. (c) Partial tumour necrosis ( 75\%) and viable tumour region. (d) Undamaged 'normal' ear tissue $\sim 2 \mathrm{~mm}$ away from the tumour edge (but still within the PDT treatment field). Central white-light photograph shows the colour-coded orientation of the two histological sections displayed for each illustrative case.

view of this, we put aside the interesting questions on the relative contributions and interplay of the three PDT mechanisms, and focused on formulating a practically useful criterion of early prediction of PDT success based on OCA evaluation of the vascular response.

In the present study, vascular reaction to PDT was analyzed on CT-26 mice ear tumour model. Relatively low thickness of tumour-bearing murine ear preparation allows for well-controlled PDT delivery and thorough OCT/OCA assessment monitoring that visualizes microstructure and blood microcirculation throughout the 1-1.5 mm depth. Further, the presence of inherently co-registered microstructural and microvascular images enables 3D geometrical assignment of the microvessels to different anatomical regions of the tissue, in particular inside and outside tumour borders ${ }^{29}$. Such microvascular segmentation was considered important, for example in the previous study ${ }^{14}$ that evaluated perfused vessels only inside the tumour (vessels in the $3 \mathrm{D}$ region occupied by microstructural-OCT-segmented tumour volume).

It was shown that although analysis of perfused vessels inside the tumour alone can reliably predict PDT failures (if there is non-negligible persisting local perfusion $24 \mathrm{hrs}$ post-treatment), this is insufficient to robustly estimate PDT successes (lack of local tumour perfusion may, but sometimes may not indicate a responder) ${ }^{14}$. It was then assumed that preserved perfusion of peri-tumourous vessels facilitates treatment failure/relapse, a supposition that was closely examined in this larger animal cohort study. And indeed, we showed that adding perfusion information from the near-tumour zone (up to $\sim 2 \mathrm{~mm}$ from the OCT-segmented pathology edge) to the local tumour microvascular response significantly improved treatment outcome prediction. Using the OCA data at $t=24$ hours after PDT, quantifying the perfusion images via the relatively simple PVD metric, and devising simple response criteria (graphically summarized in Fig. 4) yielded correct outcome prediction in all examined cases $(\mathrm{n}=31)$. The following practical and robust OCA-based criterion of PDT success was formulated: there should be no visibly perfused vessels on OCA images inside the tumour borders, and the PVD should not exceed 1\% from OCA image size in the $2 \mathrm{~mm}$ near-tumour proximity regions. In contrast to methods based on estimations of PDT procedure parameters, such as singlet oxygen concentration ${ }^{10,13}$ or evaluation of the degree of sensitizer photobleaching ${ }^{12}$, the proposed method directly evaluates the post-PDT reaction of the tissue itself, thus allowing for reliable prediction of the final PDT outcomes.

The proposed criterion and controllable ear tumour model may prove useful for fast and accurate PDT evaluation when new photosensitizers or new PDT regimes are tested. Concerning future clinical applications, OCA imaging could be applied for monitoring PDT efficacy of tumour treatments, for example in patients with basal cell carcinoma (BCC), by offering noninvasive label-free 3D imaging of vascular response shortly after the therapy (hours to 1-2 days). In the case of weak or insufficient vascular response, corrections to the treatment protocol could be enacted. We have recently obtained encouraging preliminary data on a clinical pilot study of OCA prognosis in PDT treatments of BCC. This work is continuing and will be presented in future publications. OCA monitoring of tumour and peri-tumourous blood perfusion may prove useful in adjusting PDT regime to achieve a reasonable compromise between complete tumour response and minimal surrounding tissue damage. 


\begin{abstract}
Materials and Methods
Animals and tumour model. The study was carried out on BALB/c mice $(n=31$, purchased from Nursery for Laboratory Animals, Pushino, Russian Federation). Murine colon carcinoma CT-26 cells were implanted subcutaneously in auricle tissues at the dose $2 \times 10^{5}$ cells $/ 20 \mu \mathrm{l}$ of phosphate buffered saline. The tumours grew for 13 days till visually reaching $\sim 3.5-4 \mathrm{~mm}$ diameter, then PDT was performed (described below). All animal experiments were conducted in accordance with the European Convention for the Protection of Vertebrate Animals used for Experimental and Other Scientific Purposes (ETS 123), and The Guide for the Care and Use of Laboratory Animals, (8th edition NRC 2011, National Academic Press). The experimental protocol was approved by the Research Ethics Board of the Nizhny Novgorod State Medical Academy.
\end{abstract}

Photodynamic therapy. The animals received intravenous injection of the clinically-approved photosensitizer Photoditazin (N-dimethylglucamine salt of Chlorine E6, Veta-Grand, Russia) into the tail vein at a dose of $5 \mathrm{mg} /$ $\mathrm{kg}$ body weight. One hour after photosensitizer injection, PDT was carried out. Tumours were exposed to $658 \mathrm{~nm}$ light (total irradiance $100 \mathrm{~J} / \mathrm{cm}^{2}$, irradiance rate $100 \mathrm{~mW} / \mathrm{cm}^{2}, 1,000 \mathrm{sec}$ irradiation time). This "mild" PDT regime was intentionally chosen to yield both responders and non-responders. Laser beam spot covered the tumour node plus $2 \mathrm{~mm}$ margin. During PDT delivery, the animals were anesthetized with Zoletil 50 and Rometar 2\% (similar to Xylazin) intramuscularly, as per REB-approved protocol. Although the PDT protocol was reproduced as accurately as possible, evidently natural variability in the density of vessels in the irradiated zone (important for the used mostly vasculature-targeted PDT), as well as rates of the sensitizer accumulation in the tumour and release from the vessels, reactions of the immune system, etc. resulted in occurrence of both responders and non-responders.

Although the degree of "mild" PDT results may be quantified in terms of continuous parameters (percentage of viable tumour cells, coefficient of tumour growth inhibition14, etc.), this study is focused on finding an early and accurate predictor of non-responders and responders. The formulation of such a reliable binary-type predictor is of key importance in the clinical practice of PDT.

Optical coherence angiography. OCA images were collected before PDT, immediately after $(0 \mathrm{~h}), 5 \mathrm{hrs}$ and $24 \mathrm{hrs}$ post PDT from the tumour center and at four neighboring areas of peri-tumourous margin irradiated by laser near the tumour border (up, down, right, left). All imaged tissue regions starting from $2 \mathrm{~mm}$ from the visual tumour border were considered as peri-tumoural in the study, thus defining the peritumour ROI for subsequent analysis. Three-dimensional blocks of OCT data, 256 pixels in depth $(2 \mathrm{~mm}$ in air $)$ and $1024 \times 1024$ pixels laterally (field of view $2.4 \times 2.4 \mathrm{~mm}^{2}$ ) were acquired in 26 seconds, and 3D microvascular maps were generated in real time during the acquisition process ${ }^{29}$ (Fig. 6). Before applying the angiography processing, bulk tissue motions effects were mitigated by the interframe phase difference compensation of complex-valued A-scans; further processing utilized complex-valued high-pass filtering technique previously described $\mathrm{in}^{21,30}$. In order to separately analyze vascular network inside the tumour and in peri-tumoural tissue, all OCT volumes were segmented into normal and pathological regions as described previosuly ${ }^{29}$. Briefly summarizing this automatic segmentation approach, we increased its throughput by building a machine learning model. Every pixel in the OCT structural cross-sectional image was represented as a set of decomposition coefficients of the local reflectivity profile onto two orthogonal bases. Each basis was constructed from the set of profiles for normal and pathological OCT image regions, manually segmented by a pathology expert, using Principal Component Analysis (PCA). Another set of manually segmented images was used to train the Random Forest Trees classification algorithm. More details on the algorithm parameters selection and its validation can be found in $^{29}$. For more robust analysis and quantification of the OCA images, the 3D microvascular networks (and microstructurally-segmented tumour volumes) were converted to $2 \mathrm{D}$ by projecting the whole imaged depth $(\sim 1 \mathrm{~mm})$ onto the plane ${ }^{29}$. The choice of $2 \mathrm{D}$ analysis helps to minimize the influence of shadow artifacts cast by flowing blood below the true vessels ${ }^{31}$. We emphasize that our implementation of the OCA method visualizes only perfused vessels (i.e., those exhibiting flow, rather than Brownian motion present in stagnant or anastomosed vessels; see Supplementary Information for details). The total variation denoising algorithm ${ }^{32}$ was then applied to the obtained $2 \mathrm{D}$ vascular images to reduce the noise impact, after which the vessels were binarized by simple thresholding. The threshold was set as $3 \mathrm{X}$ the standard deviation (SD) of the OCA image noise before the low-pass filtering. The criteria for such selection was best visual match between the photo of the vasculature and its appearance on the OCA images. Binary images were then skeletonized to further minimize the influence of distortions caused by projected shadows and thicker perfused vessels. Perfused vessel density (PVD) was chosen as a main metric due to its simplicity, robustness, and easy interpretation. PVD was calculated as the number of pixels of all vessel skeletons in the analyzed image area, divided by the total number of pixels in this area. Projection of the OCT-obtained tumour volume onto the same plane allows for vessel network segmentation as lying inside or outside the tumour volume, and facilitates the study of peri-tumourous perfusion influence on the efficiency of PDT.

Statistical analysis. Due to relatively small number of experimental animals (31) in this study, the threshold determination was performed on the entire data set rather than dividing it into training and validation subsets. Experimentally obtained PVD values were sorted for each scenario (each time point and type of vessels, i.e., in-tumour or peri-tumourous) and candidate threshold values were set at the midpoints between neighboring values. Then each threshold was tested in terms of diagnostic accuracy (i.e. number of correctly predicted outcomes) and the optimal threshold value was determined. The so-obtained threshold values for each prediction scenario are given in Table 1.

Next, the prediction accuracies (confidence intervals summarized in Table 1) for the proposed criteria were evaluated using the binomial-proportion-based $95 \%$ Wilson score interval ${ }^{33,34}$. The Wilson score method for confidence interval estimation is considered robust and appropriate for usage with moderate sample sizes, as applicable to our study. The statistical calculations were performed using StatsModels package for Python. 


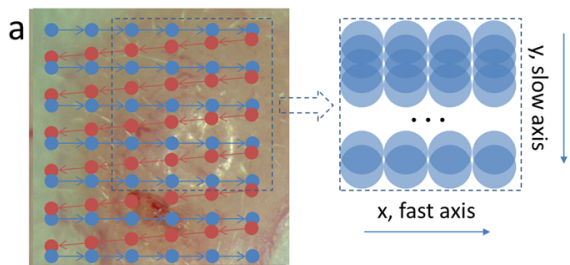

OCT-scanned volume

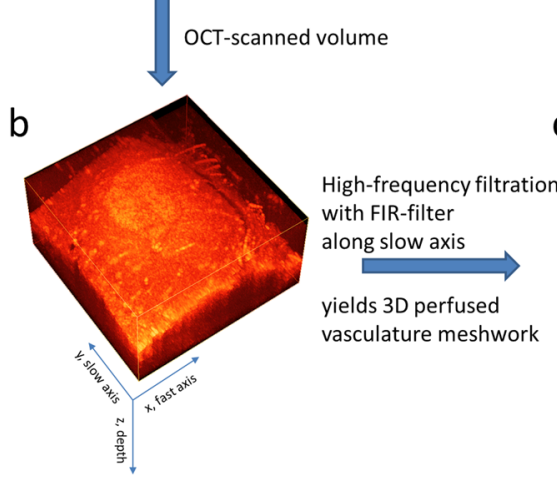

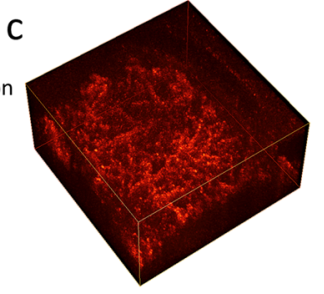

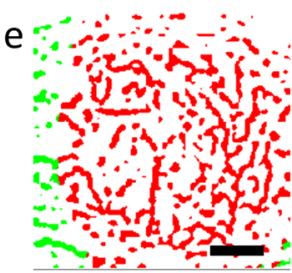

Tumour segmentation

Figure 6. OCT/OCA data acquisition and signal processing overview. (a) white light image of the tumourbearing murine ear, with superimposed scanning pattern; colored points represent individual positions of the scanning beam, scanning locations corresponding to the opposite scanning directions are represented in different colors (blue and red); only data acquired during one-directional scanning (from left to right, blue dots on the schematic) were used for OCA processing. Note the absence of repeated scanning at the same position, unlike many OCA methods. Inset shows the details of scanning beam cross sections overlap; (b) 3D OCT volume $2.4 \times 2.4 \times 1.5 \mathrm{~mm}$; c - 3D OCA volume constructed using high-frequency finite-impulse response filtering (FIR-filter) of the 3D image along the slow axis ${ }^{21} ; \mathrm{d}-$ en face Maximum Intensity Projection of the volume represented in (c); e - en face microvascular image after tumour segmentation ${ }^{29}$ : vessels inside the tumour are red and peri-tumourous vessels are green. In (d,e), scale bars are $0.5 \mathrm{~mm}$.

Histology. Histological verification of the vascular and cellular damages was carried out 7 days post PDT. Animals were sacrificed and tumour nodes were resected. Histological preparations were stained with hematoxylin and eosin (H\&E). Multiple consecutive 7- $\mu \mathrm{m}$-thick ear sections were prepared, including tumour and peri-tumourous tissue irradiated by PDT laser. Histological preparations were examined with light microscopy on Leica DM1000 system. Tumours with necrosis exceeding 95\% were considered as responders to PDT treatment. The quantitative assessment of morphological changes was undertaken via direct cell counting using the grid plate on a Leica DM1000 microscope. Under 40x magnification (field of view FOV $=326 \times 245 \mu \mathrm{m}$ ), the number of grid squares containing the necrotic cells were counted. 7-10 of such FOVs to cover the all tumour tissue were analyzed from the one cross-section. From each tumour, 3-5 cross-sections were examined. The result was presented as a percentage of the total number of grid squares, containing necrotic cells.

\section{Data Availability}

The datasets generated during and/or analyzed during the current study are available from the corresponding author on reasonable request.

\section{References}

1. Cabete, J. et al. Long-term recurrence of nonmelanoma skin cancer after topical methylaminolevulinate photodynamic therapy in a dermato-oncology department. Brazilian Annals of Dermatology 90(6), 846-850 (2015).

2. Gangloff, P. et al. Photodynamic therapy as salvage treatment for recurrent head and neck cancer. Médecine Buccale Chirurgie Buccale 18, 325-331 (2012).

3. Lindberg-Larsen, R., Sølvsten, H. \& Kragballe, K. Evaluation of recurrence after photodynamic therapy with topical methylaminolaevulinate for 157 basal cell carcinomas in 90 patients. Acta Dermato-Venereologica 92, 144-147 (2012).

4. Fantini, F. et al. Photodynamic therapy for basal cell carcinoma: clinical and pathological determinants of response. Journal of the European Academy of Dermatology and Venereology 25, 896-901 (2011).

5. Castano, A. P., Demidova, T. N. \& Hamblin, M. R. Mechanisms in photodynamic therapy: part one - photosensitizers, photochemistry and cellular localization. Photodiagnosis and Photodynamic Therapy 1(4), 279-93 (2004).

6. Allison, R. R. \& Moghissi, K. Photodynamic therapy (PDT): PDT mechanisms. Clinical Endoscopy 46, 24-29 (2013).

7. Philipp, C. M., Ziolkowska, M., Mueller, U., Urban, P. \& Berlien, H. P. Clinical adjusted photodynamic therapy. Optik Photonik 8(1), $42-44(2013)$.

8. Wan, M. T. \& Lin, J. Y. Current evidence and applications of photodynamic therapy in dermatology. Clinical, Cosmetic and Investigational Dermatology 7, 145-163 (2014).

9. Wilson, B. C. \& Patterson, M. S. The physics, biophysics and technology of photodynamic therapy. Physics in Medicine and Biology 53, R61-R109 (2008).

10. Weston, M. A. \& Patterson, M. S. Monitoring oxygen concentration during photodynamic therapy using prompt photosensitizer fluorescence. Physics in Medicine and Biology 58(20), 7039 (2013). 
11. Boere, I. A. et al. Protoporphyrin IX fluorescence photobleaching and the response of rat Barrett's esophagus following 5-aminolevulinic acid photodynamic therapy. Photochemistry and Photobiology 82(6), 1638-44 (2006).

12. Sheng, C., Hoopes, P. J., Hasan, T. \& Pogue, B. W. Photobleaching-based dosimetry predicts deposited dose in ALA-PpIX PDT of rodent esophagus. Photochemistry and Photobiology 83(3), 738-48 (2007).

13. Wilson, B. C., Patterson, M. S. \& Lilge, L. Implicit and explicit dosimetry in photodynamic therapy: a New paradigm. Lasers in Medical Science 12(3), 182-99 (1997).

14. Sirotkina, M. A. et al. Photodynamic therapy monitoring with optical coherence angiography. Scientific Reports 7, 41506, https://doi. org/10.1038/srep41506 (2017).

15. Baran, T. M. \& Foster, T. H. Fluence Rate-Dependent Photobleaching of intratumorally-administered Pc 4 Does Not Predict Tumor Growth Delay. Photochemistry and Photobiology 88(5), 1273-1279 (2012).

16. Chen, Z. et al. Noninvasive imaging of in vivo blood flow velocity using optical Doppler tomography. Optics Letters 22(14), 1119-1121 (1997).

17. Mariampillai, A. et al. Speckle variance detection of microvasculature using swept-source optical coherence tomography. Optics Letters 33(13), 1530-1532 (2008).

18. Yu, L. \& Chen, Z. Doppler variance imaging for three-dimensional retina and choroid angiography. Journal of Biomedical Optics 15(1), $016029(2010)$.

19. Zhang, A., Zhang, Q., Chen, C. L. \& Wang, R. K. Methods and algorithms for optical coherence tomography-based angiography: a review and comparison. Journal of Biomedical Optics 20(10), 100901 (2015).

20. Zaitsev, V. Y. et al. Recent trends in multimodal optical coherence tomography. II. The correlation-stability approach in OCT elastography and methods for visualization of microcirculation. Radiophysics and Quantum Electronics 57(3), $210-225$ (2014).

21. Moiseev, A. et al. OCT-based angiography device with real-time angiography B-scans visualization and hand-held probe for everyday clinical use. Journal of Biophotonics e201700292 (2018).

22. Maslennikova, A. V. et al. In-vivo longitudinal imaging of microvascular changes in irradiated oral mucosa of radiotherapy cancer patients using optical coherence tomography. Scientific Reports 7, 16505, https://doi.org/10.1038/s41598-017-16823-2 (2017).

23. Mallidi, S., Watanabe, K., Timerman, D., Schoenfeld, D. \& Hasan, T. Prediction of Tumor Recurrence and Therapy Monitoring Using Ultrasound-Guided Photoacoustic Imaging. Theranostics 5(3), 289-301 (2015).

24. Standish, B. A. et al. Interstitial Doppler optical coherence tomography as a local tumor necrosis predictor in photodynamic therapy of prostatic carcinoma: an in vivo study. Cancer Research 68(23), 9987-9595 (2008).

25. Dougherty, T. J. et al. Photodynamic Therapy. Journal of the National Cancer Institute 90(12), 889-905 (1998).

26. Hall, A. P. The role of angiogenesis in cancer. Comparative Clinical Pathology 13(3), 95-99 (2005).

27. Hanahan, D. \& Weinberg, R. A. Hallmarks of cancer: the next generation. Cell 144(5), 646-674 (2011).

28. Katsuyoshi, H., Hirotoshi, A., Hiroi, N., Akira, S. \& Yasuyuki, T. Prevention of cancer recurrence in tumor margins by stopping microcirculation in the tumor and tumor-host interface. Cancer Science 105(9), 1196-1204 (2014).

29. Moiseev, A. et al. Pixel classification method in optical coherence tomography for tumor segmentation and its complementary usage with OCT microangiography. Journal of Biophotonics 11(4), e201700072, https://doi.org/10.1002/jbio.201700072 (2018).

30. Matveev, L. A. et al. Hybrid M-mode-like OCT imaging of three-dimensional microvasculature in vivo using reference-free processing of complex valued B-scans. Optics letters 40(7), 1472-1475 (2015).

31. Jia, Y. et al. Quantitative optical coherence tomography angiography of choroidal neovascularization in age-related macular degeneration. Ophthalmology 121(7), 1435-1444 (2014).

32. Chambolle, A. An algorithm for total variation minimization and applications. Journal of Mathematical imaging and vision 20(1-2), 89-97 (2004).

33. Brown, L. D., Cai, T. T. \& DasGupta, A. Interval estimation for a binomial proportion. Statistical Science 16(2), 101-117 (2001).

34. Altman, D., Machin, D., Bryant, T. \& Gardner, M. (Eds). Statistics with confidence: confidence intervals and statistical guidelines. 2nd ed. London. BMJ Books, 254 pp. (2000).

\section{Acknowledgements}

The study has been financially supported by the Russian Science Foundation (Grant \# 14-15-00646). The method for OCT images analysis and quantification was supported by Russian Foundation for Basic Research project No.16-32-60178 mol_a_dk (AAM). The authors are grateful to Dr Marina V. Shirmanova for valuable contribution to the discussions.

\section{Author Contributions}

M.A.S., N.D.G. and A.V. designed the study. M.A.S., V.V.E. collected the experimental data. G.V.G. developed OCT setup. OCA image postprocessing was done by L.A.M., V.Y.Z., A.A.M. and S.Y.K. The results were interpreted by M.A.S., S.S.K., A.V., E.V.Z., N.D.G., F.I.F. M.A.S., A.A.M., L.A.M., V.Y.Z. and A.V. wrote the manuscript. M.A.S., A.A.M. and V.V.E. prepared the figures. All authors reviewed the manuscript.

\section{Additional Information}

Supplementary information accompanies this paper at https://doi.org/10.1038/s41598-019-43084-y.

Competing Interests: The authors declare no competing interests.

Publisher's note: Springer Nature remains neutral with regard to jurisdictional claims in published maps and institutional affiliations.

(c) (i) Open Access This article is licensed under a Creative Commons Attribution 4.0 International

License, which permits use, sharing, adaptation, distribution and reproduction in any medium or format, as long as you give appropriate credit to the original author(s) and the source, provide a link to the Creative Commons license, and indicate if changes were made. The images or other third party material in this article are included in the article's Creative Commons license, unless indicated otherwise in a credit line to the material. If material is not included in the article's Creative Commons license and your intended use is not permitted by statutory regulation or exceeds the permitted use, you will need to obtain permission directly from the copyright holder. To view a copy of this license, visit http://creativecommons.org/licenses/by/4.0/.

(c) The Author(s) 2019 